\title{
АЛГОРИТМ ПРЕОБРАЗОВАНИЯ ЭЛЕКТРОМАГНИТНОГО ПОЛЯ, ИЗМЕРЕННОГО В БЛИЖНЕЙ ЗОНЕ АНТЕННЫ НА СФЕРИЧЕСКОЙ ПОВЕРХНОСТИ, В ДАЛЬНЮЮ ЗОНУ, ОСНОВАННЫЙ НА ПРЯМОМ ВЫЧИСЛЕНИИ ФОРМУЛ СТРЭТТОНА И ЧУ
}

\author{
Н. В. АНЮТИН, К. И. КУРБАТОВ, И. М. МАЛАЙ, М. А. ОЗЕРОВ \\ Всероссийский научно-исследовательский институт физико-технических \\ и радиотехнических измерений, \\ Россия, Менделеево, Солнечногорский р-н, Московская обл.
}

\begin{abstract}
Аннотация. В статье рассмотрена возможность прямого вычисления векторных форм интеграла Кирхгофа в алгоритмах преобразования электромагнитного поля гармонического излучения антенн из ближней зоны в дальнюю зону. Для сферической схемы сканирования электромагнитного поля в ближней зоне предложен простой алгоритм на основе интеграла, полученного из формул Стрэттона и Чу. С помощью математического моделирования исследованы методические погрешности предложенного алгоритма, обусловленные сделанными при его выводе допущениями. Полная погрешность оценивается в экспериментах по восстановлению амплитудных диаграмм направленности антенн. Для сравнения во всех экспериментах приведены результаты работы классического алгоритма, основанного на разложении электрического поля по сферическим модам. Показано, что в сравнении с ним точность предложенного алгоритма не хуже, сложность программирования меньше, а скорость выполнения больше при условии восстановления диаграммы направленности только в главных сечениях.
\end{abstract}

Ключевые слова: антенные измерения; диаграмма направленности; ближняя зона; амплитудно-фазовое распределение; сферическое сканирование

\section{ВВЕДЕНИЕ}

Для прямых измерений характеристик направленности антенн требуются измерительные комплексы, обеспечивающие для излучаемых электромагнитных волн условия, эквивалентные дальней зоне (ДЗ). С увеличением электрических размеров исследуемых антенн возрастает минимальное расстояние, соответствующее условиям ДЗ, что приводит к увеличению размеров измерительного комплекса и его удорожанию. Для таких условий широкое распространение получили антенные измерительные комплексы ближней зоны (БЗ), размеры которых определяются преимущественно габаритами исследуемых антенн. Характеристики направленности антенн в таких комплек- сах измеряются косвенно с помощью алгоритмов преобразования электромагнитного поля из БЗ в ДЗ (БЗ-ДЗ алгоритмы).

Все известные БЗ-ДЗ алгоритмы основываются на различных формах интеграла Кирхгофа, который является выражением для электромагнитного поля в свободном пространстве через его известные значения на произвольной замкнутой поверхности $S_{m}$, охватывающей все источники. Существует множество эквивалентных записей интеграла Кирхгофа в скалярной и векторной формах, однако для упрощения последующего анализа воспользуемся наиболее наглядной из них - формулами Стрэттона и Чу в гауссовой симметричной системе единиц [1]: 


\section{БИБЛИОГРАФИЧЕСКИЙ СПИСОК}

1. Потехин, А.И. Некоторые задачи дифракиии электромагнитных волн. М.: Сов. радио, 1948.

2. Yaghjian, A. "An overview of near-field antenna measurements," IEEE Trans. Antennas Propag., Vol. 34, No. 1, p. 30-45, 1986. DOI: 10.1109/TAP.1986.1143727.

3. Boesman, B.; Pissoort, D.; Gielen, G.; Vandenbosch, G. A. E. "Fast and efficient near-field to near-field and near-field to far-field transformation based on the spherical wave expansion," Proc. of IEEE Int. Symp. on Electromagnetic Compatibility, EMC, 16-22 Aug. 2015, Dresden, Germany. IEEE, 2015, p. 529-534. DOI: 10.11 09/ISEMC.2015.7256218.

4. D'Agostino, Francesco; Ferrara, Flaminio; Gennarelli, Claudio; Guerriero, Rocco; Migliozzi, Massimo. "Two effective approaches to correct the positioning errors in a spherical near-field-far-field transformation," Electromagnetics, Vol. 36, No. 2, p. 78-93, 2016. DOI: 10.1080/02726343.2016.1136018.

5. Neitz, Ole; Mauermayer, Raimund A. M.; Weitsch, Yvonne; Eibert, Thomas F. "A propagating plane-wave-based near-field transmission equation for antenna gain determination from irregular measurement samples," IEEE Trans. Antennas Propag., Vol. 65, No. 8, p. 4230-4238, 2017. DOI: 10.1109/TAP.2017.2712180.

6. Cornelius, R.; Heberling, D. "Spherical wave expansion with arbitrary origin for near-field antenna measurements," IEEE Trans. Antennas Propag., Vol. 65, No. 8, p. 4385-4388, 2017. DOI: $10.1109 /$ TAP.2017.2708 099 .

7. Mauermayer, R. A. M.; Eibert, T. F. "Spherical field transformation above perfectly electrically conducting ground planes," IEEE Trans. Antennas Propag., Vol. 66, No. 3, p. 1465-1478, 2018. DOI: 10.1109/TAP.2018. 2794406.

8. D'elia, G.; Leone, G.; Pierri, R.; Schirinzi, G. "New method of far-field reconstruction from Fresnel field," Electron. Lett., Vol. 20, No. 8, p. 342-343, 1984. DOI: $10.1049 / \mathrm{el}: 19840232$.

9. Petre, P.; Sarkar, T. K. "A planar near-field to far-field transformation using an equivalent magnetic current approach," IEEE Antennas Propag. Soc. Int. Symp. Dig., 18-25 Jul. 1992, Chicago, USA. IEEE, 1992, p. 1534-1537. DOI: 10.1109/APS.1992.221746.

10. Yamaguchi, Ryo; Kimura, Yasuko; Komiya, Kazuhiro; Cho, Keizo. "A far-field measurement method for large size antenna by using synthetic aperture antenna," Proc. of 3rd European Conf. on Antennas and Propagation, 23-27 Mar. 2009, Berlin, Germany. IEEE, 2009, p. 1730-1733. URI: https://ieeexplore.ieee.org/docu ment/5067950.

11. Quijano, J. L. A.; Vecchi, G. "Field and source equivalence in source reconstruction on $3 \mathrm{D}$ surfaces," PIER, Vol. 103, p. 67-100, 2010. DOI: 10.2528/PIER10 $\underline{030309 .}$.

12. Krivosheev, Yu. V.; Shishlov, A. V.; Tobolev, A. K.; Vilenko, I. L. "Fresnel field to far field transformation using sparse field samples," Proc. of Int. Conf. on Mathematical Methods in Electromagnetic Theory, 28-30 Aug. 2012, Kyiv, Ukraine. IEEE, 2012, p. 237-242. DOI: 10.1109/MMET.2012.6331237.

13. Eibert, T. F.; Kilic, E.; Lopez, C.; Mauermayer, R. A. M.; Neitz, O.; Schnattinger, G. "Electromagnetic field transformations for measurements and simulations," PIER, Vol. 151, p. 127-150, 2015. DOI: 10.2528/PIER $\underline{14121105 .}$.

14. Eibert, T. F.; Vojvodić, D.; Hansen, T. B. "Fast inverse equivalent source solutions with directive sources," IEEE Trans. Antennas Propag., Vol. 64, No. 11, p. 4713-4724, 2016. DOI: 10.1109/TAP.2016.2606405.

15. Paulus, A.; Knapp, J.; Eibert, T. F. "Phaseless near-field far-field transformation utilizing combinations of probe signals," IEEE Trans. Antennas Propag., Vol. 65, No. 10, p. 5492-5502, 2017. DOI: 10.1109/TAP.2017. 2735463.

16. Tai, C.-T. Dyadic Green Functions in Electromagnetic Theory. IEEE, 1994.

17. Бахрах, Л. Д.; Колосов, Ю. А.; Курочкин, А.П. “Определение поля антенны в дальней зоне через значения поля в ближней зоне,” Антенны, № 24, С. 3-14, 1976.

18. Silver, S. Microwave Antenna Theory and Design, Book 19. IET, 1984. DOI: 10.1049/PBEW019E.

19. Brown, J. "A theoretical analysis of some errors in aerial measurements," Proc. IEE - Part C: Monographs, Vol. 105, No. 8, p. 343-351, 1958. DOI: 10.1049/pi-c. 1958.0044 .

20. Lee, Jeong-Seok; Song, Tae-Lim; Du, Jin-Kyoung; Koo, Tae-Wan; Yook, Jong-Gwan. "A study on near-field to far-field transformation using Stratton-Chu formula," J. Korean Institute Electromagnetic Eng. Sci., Vol. 24, No. 3, p. 316-323, 2013. DOI: 10.5515/KJKIEES. 2013.24.3.316.

21. Ding, Yu; Lin, Yang; De-Min, Fu; Qi-Zhong, Liu. "Analysis and simulation of system phase errors in 
planar near-field measurements on ultra-low sidelobe antennas," Proc. of IEEE Int. Conf. on Ultra-Wideband, 20-23 Sept. 2010, Nanjing, China. IEEE, 2010, Vol. 1, p. 1-4. DOI: 10.1109/ICUWB.2010.5614371.

22. http://www.skard.ru/?page id=5038.

23. Gibson, W. C. The Method of Moments in Electromagnetics, 2nd ed. CRC Press, 2014. URI:
https://www.crcpress.com/The-Method-of-Moments-inElectromagnetics/Gibson/p/book/9781482235791.

24. Бахрах, Л.Д.; Кременецкий, С.Д.; Курочкин, А.П.; и др. Методы измерений параметров излучающзих систем в ближней зоне. Ленинград: Наука, 1985. 